\title{
Reiki als symptomatische Krebstherapie?
}

\author{
Eine Reiki-Behandlung ( $r e i=$ Geist, \\ Seele, $\mathbf{k i}=$ Lebensenergie) soll das \\ allgemeinen Wohlbefinden steigern, \\ die Gesundheit erhalten und die \\ Selbstheilungskräfte im Krankheits- \\ fall aktivieren.
}

- Amerikanische Forscher führten eine randomisierte Studie mit 54 Prostatakrebspatienten durch. Sie erhielten zusätzlich zu einer Radiotherapie entweder acht Wochen lang Reiki ( 2 x/Woche) oder Entspannungsübungen $(1 \mathrm{x} /$ Woche) oder keine derartige Zusatzbehandlung.

Bei Behandlungsende zeigten sich keine signifikanten Differenzen zwischen der Reiki- und der Entspannungstherapiegruppe. Post-hoc-Analysen fanden jedoch positive Effekte sowohl für Reiki als auch für die Entspannungsübungen. Die Autoren folgern, dass eine "Relaxation Response“ das Wohlbefinden und die Ängstlichkeit bei Studienteilnehmern verbessert.

\section{Kommentar}

Reiki ist eine Art Geistheilung, die insbesondere von Krankenschwestern häufig appliziert wird. Die Studie scheint erstaunlich vielversprechende Ergebnisse für diese wenig plausible Behandlungsform zu liefern. Wie so häufig bei solchen Untersuchungen, könnte der Grund in erheblichen methodischen Schwächen des Studiendesigns liegen. Besonders auffällig ist die Tatsache, dass in der Reikigruppe insgesamt 16, in der Entspannungsgruppe acht und in der Kontrollgruppe null Therapeutenkontakte stattfanden. Wenn wir davon ausgehen, dass solche Kontakte anxiolytische Effekte haben, die sich auf das Wohlbefinden der Patienten positiv auswirken, dann haben wir hier bereits eine äuBerst plausible Erklärung für die beobachteten Effekte. Übernatürliche Kräfte der Geistheilung müssen also nicht als Ursache herangezogen werden. Fazit: Bei Studien zur Geistheilung und ähnlich abstrusen Behandlungsformen lohnt es sich stets, das "Kleingedruckte" kritisch zu lesen.

E. ERNST .

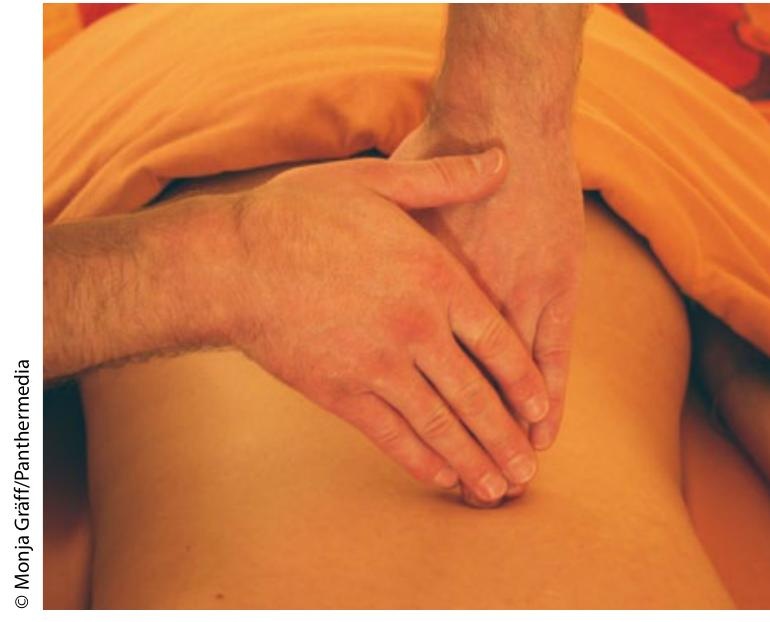

Reiki: Heilende Lebensenergie vom Behandler.

\section{Strahlenproktitis}

Ein 70-jähriger Mann stellte sich mit einer seit zwei Monaten bestehenden intermittierenden Hämatochezie vor. Zehn Jahre zuvor war er über sechs Monate hinweg wegen eines Prostatakarzinoms bestrahlt worden. Die Gesamtdosis der Bestrahlung betrug 64 Gy, und der Patient wurde anschließend als geheilt entlassen.

— Die physikalische Untersuchung des Abdomens erbrachte keine nennenswerten Befunde. Bei der rektalen Untersuchung fanden sich Blutkoagel am Fingerling. Rektoskopisch sah man eine leicht verletzliche Mukosa des Rektums mit Neigung zu Kontaktblutungen.

Bei der Kolonoskopie zeigten sich zahlreiche Teleangiektasien der Rektumschleimhaut. Bei einem entsprechenden histologischen Bild wurde eine Strahlenproktitis diagnostiziert und der Patient mit einer Argonbeamer-Behandlung the-

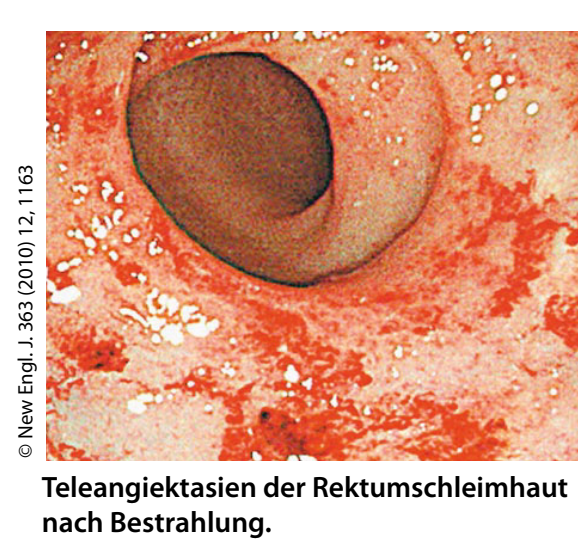

rapiert. Im Verlauf der folgenden 15 Monate Nachbeobachtung kam es nur noch zu geringfügigen rektalen Blutabgängen.

Die Strahlenproktitis ist eine weitgehend unvermeidliche Nebenwirkung einer Strahlenbehandlung im Bereich des kleinen Beckens, vor allem wegen Prostatakarzinom. Sie beinhaltet nicht nur eine Blutungsneigung, sondern gelegentlich auch Veränderungen der Stuhlgewohnheiten und Probleme bei der Defäkation. Aufgrund der weitgehenden Atrophie der Rektumschleimhaut ist eine ursächliche Behandlung nicht möglich und man muss sich auf symptomatische Maßnahmen bzw. Versuche der Blutstillung erweiterter Gefäße beschränken. H. S. FÜESSL =

\footnotetext{
- H. Lak Lee

(Hanyang University Hospital Seoul, South Korea,e-mail: alwayshang@hanyang.ac.kr): Radiation proctitis. New Engl. J. 363 (2010) 12,1163
} 